\title{
Durvalumab after chemoradiotherapy in stage III non-small cell lung cancer
}

\author{
Pascale Tomasini $^{1,2}$, Laurent Greillier ${ }^{1,2}$, Arnaud Boyer ${ }^{1,2}$, Arnaud Jeanson ${ }^{1,2}$, Fabrice Barlesi $^{1,2}$ \\ ${ }^{1}$ Aix Marseille University, Assistance Publique Hôpitaux de Marseille, Multidisciplinary Oncology \& Therapeutic Innovations dpt, Marseille, \\ France; ${ }^{2}$ Predictive Oncology Laboratory, Centre de Recherche en Cancérologie de Marseille, Inserm UMR1068, CNRS UMR7258, Aix-Marseille \\ Université UM105, Marseille, France \\ Correspondence to: Pr. Fabrice Barlesi, MD, PhD. Multidisciplinary Oncology \& Therapeutic Innovations dpt, Chemin des Bourrelys, 13915 Marseille \\ Cedex, France. Email: fabrice.barlesi@ap-hm.fr. \\ Provenance: This is an invited Editorial commissioned by the Section Editor Dr. Gang Shen (The Second Affiliated Hospital Zhejiang University \\ School of Medicine, Hangzhou, China). \\ Comment on: Antonia SJ, Villegas A, Daniel D, et al. Durvalumab after Chemoradiotherapy in Stage III Non-Small-Cell Lung Cancer. N Engl J Med \\ 2017;377:1919-29.
}

Submitted Mar 22, 2018. Accepted for publication Apr 03, 2018.

doi: $10.21037 /$ jtd.2018.04.61

View this article at: http://dx.doi.org/10.21037/jtd.2018.04.61

Immune Check Point inhibitors (ICIs) have demonstrated efficacy in advanced stage solid tumors including nonsmall cell lung cancer (NSCLC), CTLA4, programmed cell death-1 (PD-1) and PD-1 ligand 1 (PD-L1) inhibitors being the most studied drugs.

Durvalumab, previously known as MEDI4736, is a fully human monoclonal IgG1 $\kappa$ antibody directed against PD-L1 (1). Durvalumab binds with high affinity and specificity to the PD-L1 receptor expressed on tumor cells. Therefore, durvalumab blocks the interaction between PD-L1 and its ligands (PD-1 and CD-80) expressed on immune cells, and restores T-cells cytotoxic function. However, PD-L1 is also expressed on effector T-cells and studies have shown that PD-L1 blocking antibodies are able to trigger the PD-L1 intracellular signaling pathway in $\mathrm{CD} 8+\mathrm{T}$ cells in addition to their role in blocking PD-L1/PD-1 interaction (2). This intracellular signaling pathway would be likely to trigger apoptosis of T-cells and therefore produce a paradoxical effect.

In the phase I study (3), durvalumab was administered IV every 2 or 3 weeks in a $3+3$ dose escalation in 26 patients with various malignancies including NSCLC. Durvalumab induced 4 partial responses and 5 additional minor responses. Moreover, durable disease control was obtained in almost half of patients. Treatment-related adverse events (AEs) occurred in $34 \%$ of all patients, with a limited toxicity of grade 1 to 2 . Side effects consisted mainly of diarrhea, fatigue, rash and vomiting.

The phase IB study of durvalumab $(10 \mathrm{mg} / \mathrm{kg}$ IV Q2W) alone in advanced NSCLC (3) confirmed its clinical significance. Overall, 304 patients were treated. The objective response rate (ORR) was $25 \%$ for patients with PD-L1 positive tumors and $6 \%$ for patients with PD-L1 negative tumors (respectively 29\%, 26\%, and $22 \%$ after first-, second- and third-line treatment in the PD-L1+ group and $11 \%, 4 \%$ and $6 \%$ in the PD L1-group). In addition, median overall survival (OS) was 17.8 months in second-line for PD-L1+ NSCLC and 8.2 months for PD-L1-NSCLC. In the third-line setting, median OS reached 13 months for PD-L1+ NSCLC and 7.1 months for PD L1-NSCLC. All grade AEs and grade $\geq 3$ AEs were reported in $57 \%$ and $10 \%$ of patients, respectively, with $5 \%$ of AEs leading to the discontinuation of treatment (mainly related to pneumonitis and colitis).

Furthermore, the ATLANTIC (4) trial assessed durvalumab (10 mg/kg Q2W) for the third-line treatment of patients with advanced PD-L1+ NSCLC. The ORR were $7.5 \%, 16.4 \%$ and $30.9 \%$ in patients with PD-L1 expression of $<25 \%,>25 \%$ and $>90 \%$, respectively. Overall, $10.2 \%$ of patients had at least grade 3 treatment-related AEs and 2.7\% had treatment-related AEs leading to discontinuation.

Initially, ICIs have been approved by the FDA (Food 
and Drug Administration) for the treatment of advanced metastatic NSCLC, and durvalumab has mostly been studies in this area. However, there is an unmet need for an extended use of ICIs to earlier-stage NSCLC. For this reason, trials are ongoing assessing durvalumab in the adjuvant or neoadjuvant setting. The IFCT 1401-BR.31 (NCT02273375) phase III trial is recruiting patients with completely resected stage IB, II or IIIA PD-L1 + NSCLC to receive durvalumab $(20 \mathrm{mg} / \mathrm{kg}$ IV Q4W) or placebo for 1 year. Durvalumab is also being evaluated in the neoadjuvant setting in association with chemotherapy in the SAKK trial (5) and as a single agent in the IFCT-1601 IONESCO trial (EUDRACT 2016-001849-15).

Furthermore, unresectable stage III NSCLC prognosis is poor after concurrent chemo-radiotherapy, with a median progression-free survival (PFS) of approximately 8 months and a 5 -year survival rate of at best $15 \%$ (6). Moreover, no significant advance has been made during the last decade in the treatment of unresectable stage III NSCLC. Previous trials with vaccines after chemoradiotherapy had disappointing results. In the START phase III trial, patients were randomized to receive tecemotide (B-BLP25), a MUC1 antigen-specific cancer immunotherapy, or placebo after chemo-radiotherapy for NSCLC. Unfortunately, no significant OS difference was found since median OS was 25.6 months with tecemotide versus 22.3 months with placebo (adjusted HR 0.88 , 0.75-1.03; $\mathrm{P}=0.123)$ (7). Nevertheless, OS was longer in the tecemotide arm in the subgroup of patients with previous concurrent chemo-radiotherapy (30.8 versus 20.6 months) and serious immune-related AEs were similar in the two arms, suggesting immunotherapy could have a role after concurrent chemo-radiotherapy for stage III NSCLC.

In addition, there is a preclinical and clinical rational for the use of ICIs with radiotherapy. Chemotherapy and radiotherapy up-regulate PD-L1 expression in tumor cells. Zhang et al. shown that chemotherapy agents could induce PD-L1 surface expression of breast cancer cells and be responsible for cancer immunoresistance (8). In the same way, Deng et al. demonstrated that PD-L1 was upregulated in the tumor microenvironment after radiotherapy, and that the administration of a PD-L1 inhibitor could enhance the efficacy of radiotherapy (9). Furthermore, an abscopal effect, defined by a significant tumor growth inhibition outside of the radiotherapy field, was observed in mice models of breast cancers treated with radiotherapy in combination with an anti-CTLA-4 antibody (10), suggesting a synergistic effect of radiotherapy and ICIs.
For these reasons, durvalumab was studied as a consolidation treatment after chemo-radiotherapy for unresectable stage III NSCLC.

The results of the PACIFIC trial (NCT02125461), assessing durvalumab as consolidation therapy in patients with stage III NSCLC, were reported by Antonia et al. (11). In this randomized, double-blind, phase III study, patients with locally advanced, unresectable NSCLC who had not progressed after concurrent chemo-radiotherapy 2 or more cycles of platinum-based chemotherapy with concurrent definitive radiation therapy ( 54 to $66 \mathrm{~Gy}$ ) were randomized, in a 2:1 ratio, to receive either durvalumab (at a dose of $10 \mathrm{mg} / \mathrm{kg}$ intravenously) or matching placebo, every other week for up to 12 months. The study drug was administered 1 to 42 days after the patients had received chemoradiotherapy. The co-primary endpoints were PFS, assessed by blinded independent central review, and OS. The secondary endpoints of the trial were 12- and 18-month PFS rates, the ORR, the duration of response, the time to death or distant metastasis, and safety.

Among the 713 randomized patients, 709 received consolidation therapy (473 in the durvalumab arm, and 236 in the placebo arm). A pre-planned interim analysis showed that the endpoint of PFS was met, with a hazard ratio for disease progression or death of 0.52 [ $95 \%$ confidence interval $(\mathrm{CI}), 0.42$ to $0.65 ; \mathrm{P}<0.001]$. The median PFS from randomization was 16.8 months (95\% CI, 13.0 to 18.1 ) and 5.6 months (95\% CI, 4.6 to 7.8 ) with durvalumab and placebo, respectively (11). An analysis of OS, the second coprimary endpoint, was not planned at the time of this interim analysis of PFS.

Regarding secondary endpoints, the 12-month PFS rate was $55.9 \%$ versus $35.3 \%$, and the 18 -month PFS rate was $44.2 \%$ versus $27.0 \%$, in the durvalumab arm and in the placebo arm, respectively. The benefit of durvalumab was consistently observed across subgroups, notably according to NSCLC disease stage (IIIA or IIIB), pathology and PD-L1 expression in tumor samples. Of note, PD-L1 expression was assessed on the pretreatment tumor samples (i.e., obtained before chemoradiotherapy), and not on re-biopsies performed before the consolidation therapy with durvalumab or placebo [such data would have been of great interest, as chemotherapy and radiation therapy may increase PD-L1 expression $(8,9)]$. In addition the ORR was higher $(28.4 \%$ versus $16.0 \%$; $\mathrm{P}<0.001)$, and the median duration of response was longer $(72.8 \%$ versus $46.8 \%$ of patients had an ongoing response at 18 months) with durvalumab than with placebo. Moreover the median 
time to death or distant metastasis was significantly longer in the durvalumab arm compared with the placebo arm (23.2 versus 14.6 months; $\mathrm{P}<0.001$ ).

In terms of safety, grade 3 or 4 AEs occurred in $29.9 \%$ and $26.1 \%$ of patients who received durvalumab and placebo, respectively. The most common grade 3 or $4 \mathrm{AE}$ was pneumonia ( $4.4 \%$ and $3.8 \%$, respectively). Discontinuation of the study drug because of toxicity occurred in a total of $15.4 \%$ of patients in the durvalumab arm and $9.8 \%$ of patients in the placebo arm. The most frequent AEs leading to discontinuation of treatment were pneumonitis or radiation pneumonitis (in $6.3 \%$ and $4.3 \%$, respectively) and pneumonia (in $1.1 \%$ and $1.3 \%$, respectively). In summary, Antonia and colleagues concluded that PFS was significantly longer with durvalumab than with placebo, the secondary endpoints favored durvalumab and safety was similar between the 2 arms (11).

Some comments can be made about the design of the PACIFIC trial. Among the inclusion criteria, completion of the last radiation dose had initially to occur within 1 to 14 days before randomization. After a protocol amendment, this criterion was changed to 1 to 42 days before randomization, facilitating the patients' management but generating also some heterogeneity among the study. In addition, radiation therapy doses were not homogeneous among patients enrolled in the trial, and sometimes outside the inclusion criteria $(<54$ or $>66$ Gy in $7.3 \%$ of patients). Finally, there was some heterogeneity in terms of chemotherapy schedule $(26.8 \%$ of patients received induction chemotherapy before definitive chemo-radiotherapy) and drugs combination (cisplatin or carboplatin with etoposide, vinorelbine, vinblastine, docetaxel, paclitaxel or pemetrexed). However, thanks to the stratification factors and the randomized design of the PACIFIC trial, such heterogeneity in patients' management was well balanced between the 2 arms of the study. Consequently efficacy differences remain valid. Nevertheless neither the optimal type of chemoradiotherapy, nor the optimal timing of first durvalumab infusion can be determined based on the results of the PACIFIC study. Furthermore, the optimal rhythm and duration of consolidation therapy with durvalumab is not yet defined and will deserve further evaluation.

As previously mentioned, the coprimary endpoint of PFS was met in the PACIFIC study, with a difference of almost one year in median PFS between the durvalumab and the placebo arm. One can argue that median PFS in the placebo group was relatively short (5.6 months; $95 \%$ CI, 4.6 to 7.8 ) compared with the control arms of recent phase III trials in patients with locally advanced, unresectable $\operatorname{NSCLC}(7,12)$. However, patients were randomized in the PACIFIC trial up to 42 days after completion of definitive chemotherapy, and not at the beginning of anticancer treatment. Furthermore radiological disease assessment was performed at short intervals (every 8 weeks for the first 12 months and every 12 weeks thereafter) and was independently reviewed, which may have influenced PFS results. Anyway, the benefit obtained with durvalumab consolidation therapy in terms of PFS appears as of unparalleled magnitude for patients with stage III NSCLC.

Data on OS were not mature at the time of the interim analysis. These data are eagerly awaited as stage III NSCLC is potentially curable. One can nevertheless speculate on a significant benefit of durvalumab consolidation therapy in terms of OS, based on the major impact on PFS (hazard ratio for disease progression or death of 0.52 ), the significant differences in ORR and duration of response, and the data on time to death or distance metastasis. In addition, the frequency of new lesions was $20.4 \%$ with durvalumab and $32.1 \%$ with placebo, with a lower incidence of new brain metastases with durvalumab (5.5\% versus $11.0 \%$ ) (11).

Based on the results of the PACIFIC trial (11), the FDA recently approved durvalumab for the treatment of patients with stage III, unresectable NSCLC, not progressing after chemo-radiotherapy.

To go further towards a better efficacy profile, combinations of ICIs with other immunotherapies or targeted therapies should be assessed for the treatment of unresectable stage III NSCLC in combination with or consolidation after chemo-radiotherapy. Combinations of ICIs have already showed promising results for the treatment of advanced NSCLC. A phase I study of nivolumab plus ipilimumab for first-line treatment of advanced NSCLC indeed found an ORR of $38 \%$ to $47 \%$ with a duration of response of 11.8 to 12.8 months (13). In the same way, durvalumab was studied in combination with the anti-CTLA-4 tremelimumab in the first-line setting in the MYSTIC (14) and NEPTUNE (15) trials and in the second-line setting in the ARCTIC (16) phase III trial. In the MYSTIC trial, patients are randomized to receive Durvalumab (20 mg/kg Q4W) plus Tremelimumab $(1 \mathrm{mg} / \mathrm{kg} \mathrm{Q} 4 \mathrm{~W})$ or durvalumab alone versus platinum-based chemotherapy in advanced NSCLC. In the NEPTUNE trial, patients are randomized to receive either Durvalumab (20 mg/kg Q4W) plus Tremelimumab (1 mg/kg IV Q4W for up to 4 doses) or platinum-based doublet chemotherapy. 
In the ARCTIC trial, patients are randomized to receive the combination of Durvalumab plus Tremelimumab, Durvalumab alone, Tremelimumab alone or a standard of care (randomization 3:2:2:1). The results of these trials are awaited.

Regarding consolidation treatment of unresectable stage III NSCLC after chemo-radiotherapy, a phase III study is planned to assess the combination of durvalumab with the anti-IDO1 epacadostat (EUDRACT 2017-004960-36). IDO1 inhibits antitumor cell-mediated immune responses. A phase I/II study is ongoing to assess the safety profile of this combination in metastatic solid tumors including NSCLC (study ECHO-203, NCT02318277). Preliminary results of this study showed a disease control rate of $50 \%$. The main fear when combining immunotherapies after radiation therapy is the risk of pneumonitis, including radiation-induced pneumonitis. However, the rate of grade 3 or 4 pneumonitis or radiation-induced pneumonitis was similar in the 2 arms of the PACIFIC trial $(4.4 \%$ in the durvalumab arm versus $3.8 \%$ in the placebo arm) (11).

In another hand, instead of an escalation of therapeutics and a combination of several immunotherapies, radiotherapy and chemotherapy, another option for future trials in the field of unresectable stage III NSCLC treatment could be a de-escalation. The synergistic effect of radiotherapy and durvalumab may indeed be sufficient to avoid chemotherapy and focus on radiotherapy for local control and immunotherapy for systemic control of the disease.

Moreover, the optimal scheduling and dosing of the radio-immunotherapy combination remains a challenge as numerous combinations of doses, time of administration and treatment's durations are possible and were performed in the PACIFIC trial.

Mathematical modeling should help to better define an optimal schedule among numerous possibilities via the description of biological and pharmacodynamics processes. Because there are multiple different combination of radiation therapy, immunotherapy and chemotherapy, in silico simulation could help to determine the strategies that are most likely to eventually yield clinical benefit. A mathematical model of the combination between radiotherapy and ICIs targeting the PD1/PD-L1 axis and the CTLA-4 pathway has been described and explained how synchronizing immunotherapy and radiotherapy could produce synergies (17). This work provided mathematical equations to relate plasma concentrations of nivolumab and ipilimumab, as well as doses and schedules of radiotherapy, to the immune response. This mathematical model made it possible to perform in silico simulations in order to test numerous strategies in a time and cost-effective fashion and determine the best doses and scheduling to be tested in clinical trials.

A phase 1 clinical trial based on the results of these in silico simulations is ongoing to assess the association of hypo-fractionated radiotherapy plus nivolumab alone or with ipilimumab for pretreated advanced stage NSCLC patients (EUDRACT: 2017-001198-18).

In summary, immunotherapy, and specifically Durvalumab in the present case, now provides locally advanced stage NSCLC patients with a new treatment option based on an indisputable progression free survival benefit; the OS benefit linked to Durvalumab as well as the results of ongoing trials in this setting are therefore eagerly awaited.

\section{Acknowledgements}

None.

\section{Footnote}

Conflicts of Interest: Fabrice Barlesi has received personal fees and research support from Astra-Zeneca. The other authors have no conflicts of interest to declare.

\section{References}

1. Ibrahim R, Stewart R, Shalabi A. PD-L1 blockade for cancer treatment: MEDI4736. Semin Oncol 2015;42:474-83.

2. Liu X, Wu X, Cao S, et al. B7-H1 antibodies lose antitumor activity due to activation of p38 MAPK that leads to apoptosis of tumor-reactive CD8(+) T cells. Sci Rep 2016;6:36722.

3. Antonia S, Goldberg SB, Balmanoukian A, et al. Safety and antitumour activity of durvalumab plus tremelimumab in non-small cell lung cancer: a multicentre, phase $1 \mathrm{~b}$ study. Lancet Oncol 2016;17:299-308.

4. Garassino M, Vansteenkiste J, Kim JH, et al. Durvalumab in $\geq 3$ rd-Line Locally Advanced or Metastatic, EGFR/ ALK Wild-Type NSCLC: Results from the Phase 2 ATLANTIC Study. J Thorac Oncol 2017;12:S10-1.

5. Rothschild SI, Zippelius A, Prince SS, et al. 129TiP: SAKK 16/14 - anti-PD-L1 antibody durvalumab (MEDI4736) in addition to neoadjuvant chemotherapy in patients with 
stage IIIA (N2) non-small cell lung cancer (NSCLC). A multicenter single-arm phase II trial. J Thorac Oncol 2016;11:S112.

6. Aupérin A, Le Péchoux C, Rolland E, et al. Meta-analysis of concomitant versus sequential radiochemotherapy in locally advanced non-small-cell lung cancer. J Clin Oncol 2010;28:2181-90.

7. Butts C, Socinski MA, Mitchell PL, et al. Tecemotide (L-BLP25) versus placebo after chemoradiotherapy for stage III non-small-cell lung cancer (START): a randomised, double-blind, phase 3 trial. Lancet Oncol 2014;15:59-68.

8. Zhang P, Su DM, Liang M, et al. Chemopreventive agents induce programmed death-1-ligand 1 (PD-L1) surface expression in breast cancer cells and promote PD-L1mediated T cell apoptosis. Mol Immunol 2008;45:1470-6.

9. Deng L, Liang H, Burnette B, et al. Irradiation and antiPD-L1 treatment synergistically promote antitumor immunity in mice. J Clin Invest 2014;124:687-95.

10. Dewan MZ, Galloway AE, Kawashima N, et al. Fractionated but not single-dose radiotherapy induces an immune-mediated abscopal effect when combined with anti-CTLA-4 antibody. Clin Cancer Res 2009;15:5379-88.

11. Antonia SJ, Villegas A, Daniel D, et al. Durvalumab after Chemoradiotherapy in Stage III Non-Small-Cell Lung Cancer. N Engl J Med 2017;377:1919-29.

12. Bradley JD, Paulus R, Komaki R, et al. Standarddose versus high-dose conformal radiotherapy with concurrent and consolidation carboplatin plus paclitaxel with or without cetuximab for patients with stage IIIA or IIIB non-small-cell lung cancer (RTOG 0617): a randomised, two-by-two factorial phase 3 study. Lancet Oncol 2015;16:187-99.

13. Hellmann MD, Rizvi NA, Goldman JW, et al. Nivolumab plus ipilimumab as first-line treatment for advanced nonsmall-cell lung cancer (CheckMate 012): results of an open-label, phase 1, multicohort study. Lancet Oncol 2017;18:31-41.

14. Peters S, Antonia S, Goldberg SB, et al. 191TiP: MYSTIC: a global, phase 3 study of durvalumab (MEDI4736) plus tremelimumab combination therapy or durvalumab monotherapy versus platinum-based chemotherapy (CT) in the first-line treatment of patients (pts) with advanced stage IV NSCLC. J Thorac Oncol 2016;11:S139-40.

15. Mok T, Schmid P, Arén O, et al. 192TiP: NEPTUNE: A global, phase 3 study of durvalumab (MEDI4736) plus tremelimumab combination therapy versus standard of care (SoC) platinum-based chemotherapy in the first-line treatment of patients (pts) with advanced or metastatic NSCLC. J Thorac Oncol 2016;11:S140-1.

16. Planchard D, Yokoi T, McCleod MJ, et al. A Phase III Study of Durvalumab (MEDI4736) With or Without Tremelimumab for Previously Treated Patients With Advanced NSCLC: Rationale and Protocol Design of the ARCTIC Study. Clin Lung Cancer 2016;17:232-6.e1.

17. Serre R, Benzekry S, Padovani L, et al. Mathematical Modeling of Cancer Immunotherapy and Its Synergy with Radiotherapy. Cancer Res 2016;76:4931-40.

Cite this article as: Tomasini P, Greillier L, Boyer A, Jeanson A, Barlesi F. Durvalumab after chemoradiotherapy in stage III non-small cell lung cancer. J Thorac Dis 2018;10(Suppl 9):S1032-S1036. doi: 10.21037/jtd.2018.04.61 